\title{
Arctic Observing Summit 2020: Conference Statement and Call to Action
}

\author{
by Maribeth Murray and Arctic Observing Summit Committee Members
}

\section{INTRODUCTION}

A RCTIC ENVIRONMENTAL CHANGE CONTINUES unabated and at a rapid pace. Sustained observations that enable us to track, understand, and project this change are essential. Observations are necessary to guide adaptation and mitigation responses from local to global scales. The Arctic Observing Summit (AOS) is a biennial event convened as part of the Sustaining Arctic Observing Networks (SAON) initiative to guide the design, coordination, and long-term operation of an international network of observing systems that improves our understanding of and response to Arctic change. The 5th AOS (30 March-2 April 2020) was set to take place in Akureyri, Iceland under the theme of "Observing for Action". Given the circumstances introduced by the coronavirus disease 2019 (COVID-19), the Summit was transformed into an online forum, drawing over 350 participants from 28 countries, including more than 40 Indigenous experts and representatives of Indigenous peoples and organizations. This Conference Statement summarizes the main conclusions of the summit and the calls to action from the participants. It will be complemented by a detailed report to be released through the AOS website (arcticobservingsummit.org) in late summer 2020.

\section{KEY CONCLUSIONS AND RECOMMENDATIONS}

1. Use SAON's Roadmap for Arctic Observing and Data Systems (ROADS) process to identify Essential Arctic Variables (EVA) and prioritized Shared Arctic Variables (SAV), identified by their importance to multiple information user groups and applications, where Arctic Indigenous collaboration is critical for the success of the process and outcome.

2. Enhance coordination of Arctic observations, including identification of gaps and integration with global observing systems, to better inform adaptation and policy responses.

3. Expand Arctic observing efforts to reflect holistic Indigenous worldviews by directing funding to local and regional activities and increase engagement of Indigenous expertise in international work.
4. Work towards a broadly networked, collaborative, and interoperable Arctic digital system based on a co-production model and ethical data principles such as Collective Benefit; Authority to Control; Responsibility; and Ethics (CARE).

\section{RESPONDING TO THE COVID-19 PANDEMIC}

The impacts of COVID-19 on all aspects of daily life now experienced across the globe may well amplify existing hardships in Arctic communities, including food insecurity and insufficient emergency response, health care, transportation and communications infrastructure. Our nations are rightfully concerned about the impacts of COVID-19 and future pandemics on Arctic Indigenous people. Travel to the Arctic presents the threat of carrying the contagion to highly vulnerable populations. Scientific researchers may be in a position to help Arctic people understand and anticipate impacts to their health, economy, environment, communities, and overall well-being. AOS participants acknowledge that tracking the course of the present crisis and exploring effective responses are critical in developing options that increase preparedness and resilience of Arctic communities in the face of future disruptions and transformative change.

The COVID-19 crisis also threatens to interrupt critical observations and collection of time series in the Arctic, requiring immediate action to minimize loss of vital data sets and the information derived from them. AOS participants recognize the leadership role of Indigenous communities who have traditional networks of observations and who may help to sustain critical long-term scientific observations and research infrastructures in Arctic communities and remote locations. Now is the time to leverage our diverse expertise and experiences to fullest advantage for broadest benefit.

\section{CALLS TO ACTION}

In light of the current global circumstances, AOS 2020 participants call for an international effort that advances and supports the development of partnerships which allow 
Arctic Indigenous communities to draw on their strengths to sustain both Indigenous- and scientific-observing efforts. The combination of observations that address Indigenous communities' priorities and operational needs with those helping maintain critical research time series, deserve particular attention.

We call for new and existing research infrastructure (e.g., research stations and vessels) to be equipped and utilized so that they may function as critical responders and support the sharing of skills and expertise, wide dissemination of information, and maintenance of observing programs even in times of crisis. Where needed, infrastructure should support remote communities by responding to their needs in times of difficulty now and into the future. Here, the Forum of Arctic Research Operators can serve as an important partner. This approach should include the use of remote-sensing data to complement and, where possible, fill in for temporary loss of in situ observations.

We call for identifying, and where necessary, creating new funding opportunities. This is an appropriate role in particular for the Arctic Funders Forum established in response to the 2nd Arctic Science Ministerial, as well as for private foundations, nonprofits, national and state agencies, and others. Funding needs to be more flexible and responsive to the needs of communities and shared research interests.

We recommend that SAON quickly establish an Expert Panel on Health and Well-being composed of Indigenous experts, knowledge holders, youth, as well as health, social, and natural scientists, global observing network organizations, and other Arctic residents. ROADS will guide the development of new partnerships with Indigenous communities towards Arctic observing system co-design and co-management approaches.

\section{BROADER CONCLUSIONS}

The following paragraphs reflect the outcomes of work conducted within the five Working Groups (WG) under AOS 2020: Design, Optimization and Implementation of the Observing System (WG1), Observing in Support of Adaptation and Mitigation (WG2), Observing in Support of Indigenous Food Security and Related Needs (WG3), Data Interoperability and Federated Search (WG4), and Arctic Observations in the context of Global Observing Initiatives (WG5).

We recognize the value and experience of global observing organizations (i.e., World Meteorological Organization, the intergovernmental partnership Group on Earth Observations, and Global Ocean Observing System). Where possible, and with a match of essential variables, global observing systems should expand more forcefully into the Arctic, at least with a subset of their observations. The Arctic observing community can add particular value to global efforts through the ROADS process by sharing requirements, resources, and information across sectors and disciplines. We recommend that in identifying EAVs, they be prioritized as SAVs, identified by their importance to multiple information user groups and applications. Arctic Indigenous community and Indigenous organization collaboration throughout the process is critical for success. Expert panels comprising observation data providers and users, Indigenous and Arctic communities prominent among these, and coordinated through SAON would be called on to define these variables. Panels would use processes established by global observing systems for identifying and defining EAVs and SAVs wherever possible and should be broadly inclusive and draw on rounds of user community input to best reflect a range of perspectives. This process should be established as soon as possible. International networks are invited to develop formal engagement mechanisms or to help lead the process where appropriate.

To guide the identification of SAVs, we recommend launching regional studies. Regions such as the Bering Sea, the Beaufort and Mackenzie Delta area, Baffin Bay and surrounding coasts, and the Barents Sea with strong regional networks of Indigenous observers, broadly international scientific activity, large-scale commercial fisheries, and major impacts from rapid environmental change are particularly well suited locales for regional efforts.

Regional and pan-Arctic efforts under ROADS need to be complemented by continued and expanded funding and infrastructure support of in situ observations and field measurements. The importance of such efforts cannot be overstated given that for example, in the Arctic Ocean, ship-borne research observations currently serve as almost the only source of accurate information.

The engagement of Arctic Council Working Groups in the ROADS process is essential and is expected to enhance outcomes from the activities outlined in this document.

Improved understanding of system components, essential variables, and processes supports our ability to project the longer-term trajectory of the system and plan for the future. Near real-time data is vital to decision-makers and informs operational and tactical decision making, as well as longer-term adaptation and mitigation. We recommend continued development of easily understood graphical data drawn from multiple observing or monitoring programs, networks, and systems to project long-term trajectories and information flow on short-time scales. This helps to minimize risk and inform planning and adaptation in a crisis situation.

Planning for, adapting to, and mitigating change in the Arctic, as elsewhere, requires the sustained and iterative design and implementation of a pan-Arctic, internationally supported network of observing systems. Many elements of the system are already in place but there are gaps to be identified and filled to maximize benefits. We recommend quickly identifying essential variables most useful for observing in support of disaster management and risk reduction, improving resilience and co-management of terrestrial and marine ecosystems including wildlife, and 
ensuring the resilience of Arctic communities and people. Indigenous and science-based observing together should inform decision making across time, space, people, and organizations. Information from such observing approaches should support development of policy, real-world solutions to existing and emerging problems, and the implementation of adaptation initiatives and mitigation efforts.

Never before has the importance of working together been amplified as in the current COVID-19 pandemic. We acknowledge the value of a paradigm shift at multiple levels to advance observing for action. Indigenous Peoples, specifically youth, need to drive, determine, and act on research priorities. Direct funding is imperative for local to regional level efforts and to strengthen communitydriven observations for action, physical and technological infrastructure, and development of best practices, protocols and critical observing needs. Therefore, ROADS mapping efforts should be expanded to reflect holistic Indigenous worldviews and a food security lens to improve decisionmaking and links among monitoring programs and management systems. 'Health, Wellness, and Wellbeing' is critical to Arctic Indigenous Peoples and should be a central theme at AOS 2022 with invited leadership from Indigenous Peoples. We need immediate, strong, and unified messaging from all leadership levels for capacity building (people, infrastructure, and systems) at regional and local levels to grow active participation in observing for action. Substantial funding is needed for many Indigenous experts, knowledge holders, and youth to engage across scaled activities (local to international), establishing regional expert panels to engage at relevant organizations and forums (e.g. Arctic Council, International Arctic Science Committee, SAON/ROADS, etc.), and a more immediate effort to include them as part of official delegations to the Third Arctic Science Ministerial.

Advancing several years of community building, AOS participants discussed data in the context of the broader Arctic observing system, while recognizing the broader, rapid evolution of data technologies and methods. Maximizing the benefit of these advancements requires that the Arctic data community work closely with the global community and the Arctic observing community to address focused, real-world problems that are important to Arctic residents, and Indigenous peoples of the Arctic in particular. Specifically, this should include the recognition of the right of Indigenous Peoples and nations to govern collection, ownership, and application of their own data, and the broad adherence of emerging principles and protocols such as the CARE principles. We need to continue to work towards a broadly networked, collaborative, interoperable Arctic digital system based on a co-production model that includes much-needed mutual education and training. We recognize the need for the necessary resources to adequately support all actors participating in the co-production model. Achieving this vision will improve access to, and reuse of, invaluable Arctic data for the benefit of all users.

Over the coming year and beyond, members of the Arctic data community will work together with Indigenous organizations and people, other Arctic stakeholders, and the SAON/ROADS process to realize a co-production model. To continue functioning well as a community, we will enhance, extend, and formalize the SAON Arctic Data Committee and its pivotal role in driving collaboration. Building on existing efforts, we will focus on establishing a distributed, co-owned, sustainable, and coherent registry of digital resources which all partners can co-develop, access, and leverage for their needs.

Maribeth Murray is the Executive Director of the Arctic Institute of North America. Email: Murraym@ucalgary.ca

Arctic Observing Summit 2020 Executive Committee Members. Email: https://arcticobservingsummit.org/executivecommittee-members- 0 